\title{
Romosozumab and blosozumab: alternative drugs of mechanical strain-related stimulus toward a cure for osteoporosis
}

\author{
Toshihiro Sugiyama*, Tetsuya Torio, Tsuyoshi Miyajima, Yoon Taek Kim and Hiromi Oda \\ Department of Orthopaedic Surgery, Saitama Medical University, Saitama, Japan
}

Keywords: osteoporosis, mechanostat, sclerostin, romosozumab, blosozumab

\section{Treat-to-Target Strategy in Osteoporosis}

In addition to other chronic diseases such as hypertension, hypercholesterolemia, and diabetes, a treat-to-target strategy was recently applied in rheumatoid arthritis and has now been discussed in osteoporosis. An important goal of osteoporosis therapy is normal risk of hip fracture associated with significant morbidity and mortality, but the anti-fracture efficacies of currently approved drugs are limited $(1,2)$. Although fundamental methods to effectively prevent osteoporotic fracture include pharmacological treatment of sarcopenia that results in improving bone fragility as well as reducing fall risk, the present article focuses on anti-sclerostin antibodies such as romosozumab and blosozumab, the investigational agents for osteoporosis, and provides new insights into their effects from natural homeostatic system in the skeleton.

\section{OPEN ACCESS}

Edited and reviewed by: Jonathan H. Tobias,

University of Bristol, UK

*Correspondence:

Toshihiro Sugiyama

tsugiym@saitama-med.ac.jp

Specialty section: This article was submitted to Bone Research, a section of the journal Frontiers in Endocrinology

Received: 15 March 2015 Accepted: 06 April 2015 Published: 21 April 2015

Citation:

Sugiyama T, Torio T, Miyajima T, Kim YT and Oda H (2015) Romosozumab and blosozumab: alternative drugs of mechanical strain-related stimulus toward a cure for osteoporosis.

Front. Endocrinol. 6:54. doi: 10.3389/fendo.2015.00054

\section{Alternative Drugs of Mechanical Strain-Related Stimulus}

The human skeleton normally responds to the change in local mechanical environment at each skeletal site to maintain resultant elastic deformation (strain) of bone; increased or decreased bone strain would induce bone gain or loss, respectively (3-5). This mechanical strain-related feedback control called the mechanostat $(6,7)$ plays a key role in the management of osteoporosis; increased bone strength by an osteoporosis drug results in decreased bone strain, indicating that the effect of osteoporosis therapy is limited by the mechanostat (Figure 1) (5). Approaches to reduce the limitation include pharmacologically enhancing skeletal response to mechanical stimulation (8), but this might not efficiently reduce the risk of fallrelated hip fracture because the skeleton is adapted to the mechanical environment resulting from habitual physical activity but not to the unusual direction of mechanical force by falls. Consequently, an ideal strategy is to develop an alternative agent of mechanical strain-related stimulus (5).

One example would be investigational anti-sclerostin antibodies such as romosozumab and blosozumab; experimental evidence has established that the production of sclerostin secreted by osteocytes is increased by skeletal disuse and decreased by skeletal loading $(9,10)$. In addition, bone formation induced by intermittent treatment with parathyroid hormone is associated with the inhibition of sclerostin (11), suggesting that teriparatide could partly have a similar effect. There is, however, an obvious difference between the effects of anti-sclerostin antibodies and teriparatide, if injected daily (8), on bone remodeling; remodeling-based, coupled bone resorption and formation are significantly promoted by the latter, but not by the former. 


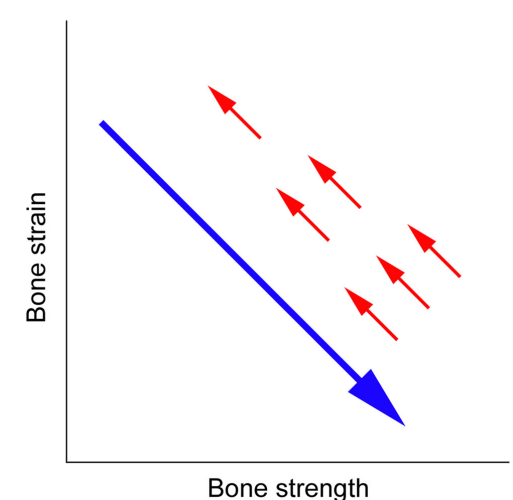

FIGURE 1 | Mechanical strain-related feedback control of bone strength. A long arrow indicates the effect of osteoporosis therapy that increases bone strength and thus decreases bone strain from physical activity, and short arrows indicate the negative feedback control of bone strength that returns bone strain to its pre-treatment level (5).

\section{Modeling-Based Effects of Anti-Sclerostin Antibodies}

In contrast to bone remodeling, modeling-based bone formation and resorption are not coupled, and mechanical stimulation is a natural uncoupling factor that stimulates bone formation and inhibits bone resorption; experimental data in skeletally mature animals $(12,13)$ show that strong suppression of bone resorption by risedronate or denosumab does not impair modeling-based bone formation induced by artificial mechanical loading or habitual physical activity. In agreement with the above suggestion that investigational anti-sclerostin antibodies such as romosozumab and blosozumab are alternative drugs of mechanical strainrelated stimulus, an experiment using male cynomolgus monkeys found a marked increase in modeling-based bone formation by romosozumab (14) and phase 2 clinical studies in postmenopausal women confirmed that both romosozumab and blosozumab treatments rapidly induced an increase in bone formation and a decrease in bone resorption $(15,16)$.

\section{Optimal Doses of Anti-Sclerostin Antibodies}

In postmenopausal women with low areal bone mineral density (BMD), romosozumab and blosozumab treatments for 1 year increased areal BMD at the lumbar spine and hip dosedependently; mean changes from baseline in areal BMD at the lumbar spine, total hip and femoral neck by romosozumab [placebo vs. highest dose ( $210 \mathrm{mg}$ every 1 month)] were -0.1 vs. $11.3 \%,-0.7$ vs. $4.1 \%$, and -1.1 vs. $3.7 \%$, respectively, while those by blosozumab [placebo vs. highest dose ( $270 \mathrm{mg}$ every 2 weeks)] were -1.6 vs. $17.7 \%,-0.7$ vs. $6.7 \%$, and -0.6 vs. $6.3 \%$, respectively $(15,16)$. Notably, however, areal BMD at the one-third radius was not changed by the highest dose of romosozumab $(-0.9$ vs. $-1.2 \%$, respectively) and non-significantly increased only by the highest dose of blosozumab ( -1.4 vs. $0.9 \%$, respectively) $(15,16)$.
The effects of anti-sclerostin antibodies at the radius appear to reflect the fact that forearm is not exposed to high levels of mechanical strain under normal physical activity. Experimental evidence that the production of sclerostin secreted by osteocytes is increased by skeletal disuse and decreased by skeletal loading $(9,10)$ suggests that the levels of sclerostin expression in nonweight-bearing bones such as the radius could be higher than those in weight-bearing bones such as the lumbar spine and hip. Consequently, it would be possible to speculate that even highest doses of romosozumab and blosozumab selected in phase 2 clinical studies were not enough for the radius. Indeed, the strongest effects on areal BMD at the lumbar spine and hip were achieved with the highest dose of blosozumab and only this regimen resulted in a trend of increase in areal BMD at the radius $(15,16)$; further higher doses of blosozumab might increase areal BMD at the radius dose-dependently. Several lines of evidence to support this hypothesis include (i) patients with sclerosteosis due to deficiency of sclerostin have higher areal BMD at the radius as well as the lumbar spine and hip (17) and (ii) appropriate doses of anti-sclerostin antibodies effectively increase bone mass in animals with skeletal disuse or unloading $(18,19)$.

If the above logic is correct, the highest doses of romosozumab ( $210 \mathrm{mg}$ every $1 \mathrm{month}$ ) and blosozumab ( $270 \mathrm{mg}$ every 2 weeks) are unlikely to cause unwanted bony overgrowth at non-weightbearing sites such as the face and skull in postmenopausal women with osteoporosis. In contrast, however, further higher doses of these drugs would be required to improve skeletal fragility in patients with reduced physical activity; one useful indicator to determine optimal doses of anti-sclerostin antibodies could be areal $\mathrm{BMD}$ at the radius.

\section{Limitation of Treatment with Anti-Sclerostin Antibodies}

Both romosozumab and blosozumab treatments in postmenopausal women with low areal BMD showed that marked changes in circulating bone formation and resorption markers returned to the pre-treatment levels within a year despite the continued treatments $(15,16)$. The existence of other mechanotransduction pathways independent of sclerostin (20) indicates that treatment with an anti-sclerostin antibody cannot escape from the mechanostat-related limitation of osteoporosis therapy (5).

The relation between circulating sclerostin and bone mass would support this theory. Sclerostin-related high bone mass in patients with sclerosteosis or van Buchem disease and heterozygous carriers of these diseases is linked to lower levels of circulating sclerostin $(21,22)$, while circulating sclerostin and bone mass in normal women and men have a positive correlation (2325 ). This discrepancy suggests that higher bone mass associated with other mechanotransduction pathways independent of sclerostin would cause lower mechanical strain in the skeleton and thus could result in compensatory higher sclerostin production according to the mechanostat, although the positive correlation between circulating sclerostin and bone mass is also influenced by the fact that higher bone mass results in more osteocytes, which are the main source of sclerostin (26). 


\section{Withdrawal of Treatment with Anti-Sclerostin Antibodies}

Results of 1-year post-treatment follow-up after 1-year treatment with blosozumab were recently reported in the phase 2 clinical trial of postmenopausal women with low areal BMD. Mean changes from baseline in areal BMD at the lumbar spine, total hip, and femoral neck by the highest dose ( $270 \mathrm{mg}$ every 2 weeks) of blosozumab (1-year treatment vs. 1-year treatment plus 1-year follow-up without treatment) were 17.7 vs. $6.9 \%, 6.7$ vs. $3.9 \%$, and 6.3 vs. $5.3 \%$, respectively (27).

The mechanostat indicates that bone strength returns to baseline after the withdrawal of treatment (5) and the speed of this reverse change depends on the drug (28-30). The above sitespecific difference in the reduction of areal BMD could partly result from less mechanical loading at the lumbar spine, possibly associated with higher sclerostin production $(9,10)$. Treatment with an anti-sclerostin antibody can reinforce the fragile skeleton by non-site-specific bone apposition, while its discontinuation

\section{References}

1. Crandall CJ, Newberry SJ, Diamant A, Lim YW, Gellad WF, Booth MJ, et al. Comparative effectiveness of pharmacologic treatments to prevent fractures: an updated systematic review. Ann Intern Med (2014) 161:711-23. doi:10.7326/ M14-0317

2. Kanis JA, McCloskey E, Branco J, Brandi ML, Dennison E, Devogelaer JP, et al. Goal-directed treatment of osteoporosis in Europe. Osteoporos Int (2014) 25:2533-43. doi:10.1007/s00198-014-2787-1

3. Christen P, Ito K, Ellouz R, Boutroy S, Sornay-Rendu E, Chapurlat RD, et al. Bone remodelling in humans is load-driven but not lazy. Nat Commun (2014) 5:4855. doi: $10.1038 /$ ncomms5855

4. Bhatia VA, Edwards WB, Johnson JE, Troy KL. Short-term bone formation is greatest within high strain regions of the human distal radius: a prospective pilot study. J Biomech Eng (2015) 137:011001. doi:10.1115/1.4028847

5. Sugiyama T, Kim YT, Oda H. Osteoporosis therapy: a novel insight from natural homeostatic system in the skeleton. Osteoporos Int (2015) 26:443-7. doi:10.1007/s00198-014-2923-y

6. Frost HM. Bone's mechanostat: a 2003 update. Anat Rec A Discov Mol Cell Evol Biol (2003) 275:1081-101. doi:10.1002/ar.a.10119

7. Meakin LB, Price JS, Lanyon LE. The contribution of experimental in vivo models to understanding the mechanisms of adaptation to mechanical loading in bone. Front Endocrinol (Lausanne) (2014) 5:154. doi:10.3389/fendo.2014. 00154

8. Sugiyama T, Torio T, Sato T, Matsumoto M, Kim YT, Oda H. Improvement of skeletal fragility by teriparatide in adult osteoporosis patients: a novel mechanostat-based hypothesis for bone quality. Front Endocrinol (Lausanne) (2015) 6:6. doi:10.3389/fendo.2015.00006

9. Robling AG, Turner CH. Mechanical signaling for bone modeling and remodeling. Crit Rev Eukaryot Gene Expr (2009) 19:319-38. doi:10.1615/ CritRevEukarGeneExpr.v19.i4.50

10. Moustafa A, Sugiyama T, Prasad J, Zaman G, Gross TS, Lanyon LE, et al. Mechanical loading-related changes in osteocyte sclerostin expression in mice are more closely associated with the subsequent osteogenic response than the peak strains engendered. Osteoporos Int (2012) 23:1225-34. doi:10.1007/ s00198-011-1656-4

11. Kramer I, Keller H, Leupin O, Kneissel M. Does osteocytic SOST suppression mediate PTH bone anabolism? Trends Endocrinol Metab (2010) 21:237-44. doi:10.1016/j.tem.2009.12.002

12. Sugiyama T, Meakin LB, Galea GL, Jackson BF, Lanyon LE, Ebetino FH, et al. Risedronate does not reduce mechanical loading-related increases in cortical and trabecular bone mass in mice. Bone (2011) 49:133-9. doi:10.1016/j.bone. 2011.03.775 would result in mechanical strain-related, site-specific bone loss. Local bone strain from normal physical activity is lower in the inner compartments, suggesting that bone loss caused by the mechanostat-related negative feedback is higher at the trabecular and endosteal surfaces. In contrast, newly formed bone at the periosteal surface through modeling-based apposition might not be resorbed because of a lack of efficient bone resorption in this region.

\section{Conclusion}

Anti-sclerostin antibodies such as romosozumab and blosozumab are the alternative drugs of mechanical strain-related stimulus that can overcome the mechanostat-related limitation of osteoporosis therapy (Figure 1) (5). It is expected that these agents will make a treat-to-target strategy in osteoporosis possible in the near future. Further studies are desired to investigate their optimal doses, especially depending on the levels of habitual physical activity, as well as appropriate duration of the treatments.

13. Ominsky MS, Libanati C, Niu QT, Boyce RW, Kostenuik PJ, Wagman RB, et al Sustained modeling-based bone formation during adulthood in cynomolgus monkeys may contribute to continuous BMD gains with denosumab. J Bone Miner Res (2015). doi:10.1002/jbmr.2480

14. Ominsky MS, Niu QT, Li C, Li X, Ke HZ. Tissue-level mechanisms responsible for the increase in bone formation and bone volume by sclerostin antibody. J Bone Miner Res (2014) 29:1424-30. doi:10.1002/jbmr.2152

15. McClung MR, Grauer A, Boonen S, Bolognese MA, Brown JP, Diez-Perez A, et al. Romosozumab in postmenopausal women with low bone mineral density. N Engl J Med (2014) 370:412-20. doi:10.1056/NEJMoa1305224

16. Recker RR, Benson CT, Matsumoto T, Bolognese MA, Robins DA, Alam J, et al. A randomized, double-blind phase 2 clinical trial of blosozumab, a sclerostin antibody, in postmenopausal women with low bone mineral density. J Bone Miner Res (2015) 30:216-24. doi:10.1002/jbmr.2351

17. Gardner JC, van Bezooijen RL, Mervis B, Hamdy NA, Lowik CW, Hamersma $\mathrm{H}$, et al. Bone mineral density in sclerosteosis; affected individuals and gene carriers. J Clin Endocrinol Metab (2005) 90:6392-5. doi:10.1210/jc. 2005- 1235

18. Tian XY, Jee WS, Li X, Paszty C, Ke HZ. Sclerostin antibody increases bone mass by stimulating bone formation and inhibiting bone resorption in a hindlimbimmobilization rat model. Bone (2011) 48:197-201. doi:10.1016/j.bone.2010. 09.009

19. Spatz JM, Ellman R, Cloutier AM, Louis L, van Vliet M, Suva LJ, et al. Sclerostin antibody inhibits skeletal deterioration due to reduced mechanical loading. J Bone Miner Res (2013) 28:865-74. doi:10.1002/jbmr.1807

20. Morse A, McDonald M, Kelly N, Melville K, Schindeler A, Kramer I, et al. Mechanical load increases in bone formation via a sclerostin-independent pathway. J Bone Miner Res (2014) 29:2456-67. doi:10.1002/jbmr.2278

21. van Lierop AH, Hamdy NA, Hamersma H, van Bezooijen RL, Power J, Loveridge $\mathrm{N}$, et al. Patients with sclerosteosis and disease carriers: human models of the effect of sclerostin on bone turnover. J Bone Miner Res (2011) 26:2804-11. doi:10.1002/jbmr.474

22. van Lierop AH, Hamdy NA, van Egmond ME, Bakker E, Dikkers FG, Papapoulos SE. Van Buchem disease: clinical, biochemical, and densitometric features of patients and disease carriers. J Bone Miner Res (2013) 28:848-54. doi:10.1002/ jbmr.1794

23. Modder UI, Hoey KA, Amin S, McCready LK, Achenbach SJ, Riggs $\mathrm{BL}$, et al. Relation of age, gender, and bone mass to circulating sclerostin levels in women and men. J Bone Miner Res (2011) 26:373-9. doi:10.1002/jbmr. 217

24. Polyzos SA, Anastasilakis AD, Bratengeier C, Woloszczuk W, Papatheodorou A, Terpos E. Serum sclerostin levels positively correlate with lumbar spinal bone mineral density in postmenopausal women - the six-month effect of 
risedronate and teriparatide. Osteoporos Int (2012) 23:1171-6. doi:10.1007/ s00198-010-1525-6

25. Szulc P, Bertholon C, Borel O, Marchand F, Chapurlat R. Lower fracture risk in older men with higher sclerostin concentration: a prospective analysis from the MINOS study. J Bone Miner Res (2013) 28:855-64. doi:10.1002/jbmr. 1823

26. Gregson CL, Poole KE, McCloskey EV, Duncan EL, Rittweger J, Fraser WD, et al. Elevated circulating sclerostin concentrations in individuals with high bone mass, with and without LRP5 mutations. J Clin Endocrinol Metab (2014) 99:2897-907. doi:10.1210/jc.2013-3958

27. Recknor CP, Recker RR, Benson CT, Robins DA, Chiang AY, Alam J, et al. The effect of discontinuing treatment with blosozumab: follow-up results of a phase 2 randomized clinical trial in postmenopausal women with low bone mineral density. J Bone Miner Res (2015). doi:10.1002/jbmr.2489

28. Boonen S, Ferrari S, Miller PD, Eriksen EF, Sambrook PN, Compston J, et al. Postmenopausal osteoporosis treatment with antiresorptives: effects of discontinuation or long-term continuation on bone turnover and fracture risk - a perspective. J Bone Miner Res (2012) 27:963-74. doi:10.1002/jbmr.1570
29. Padhi D, Jang G, Stouch B, Fang L, Posvar E. Single-dose, placebo-controlled, randomized study of AMG 785, a sclerostin monoclonal antibody. J Bone Miner Res (2011) 26:19-26. doi:10.1002/jbmr.173

30. McColm J, Hu L, Womack T, Tang CC, Chiang AY. Single- and multiple-dose randomized studies of blosozumab, a monoclonal antibody against sclerostin, in healthy postmenopausal women. J Bone Miner Res (2014) 29:935-43. doi:10. 1002/jbmr.2092

Conflict of Interest Statement: The authors declare that the research was conducted in the absence of any commercial or financial relationships that could be construed as a potential conflict of interest.

Copyright (C) 2015 Sugiyama, Torio, Miyajima, Kim and Oda. This is an open-access article distributed under the terms of the Creative Commons Attribution License (CC $B Y)$. The use, distribution or reproduction in other forums is permitted, provided the original author(s) or licensor are credited and that the original publication in this journal is cited, in accordance with accepted academic practice. No use, distribution or reproduction is permitted which does not comply with these terms. 